\title{
LOW FRICTION ARTHROPLASTY FOR OLD QUIESCENT INFECTION OF THE HIP
}

\author{
R. LAFORGIA, J. C. M. MURPHY, T. R. REDFERN \\ From Wrightington Hospital, Wigan
}

\begin{abstract}
We report the results of low friction arthroplasty in 72 old tuberculous hips and 42 hips with old quiescent septic arthritis. Follow-up was for a minimum of two years. Recrudescence of tuberculosis was seen in only one patient, but deep infection occurred in four of the hips with previous septic arthritis. The reasons for this are discussed.

It is recommended that patients with old tuberculous hips should have a course of anti-tuberculous drugs before operation, that patients with previous septic infection should have prophylactic antibiotics and antibiotic-loaded cement and that the operation should be performed by an experienced surgeon.
\end{abstract}

Hips affected by septic arthritis frequently become osteoarthritic, or fused. Tuberculous infection also may lead to irrevocable damage to the articular surfaces with secondary osteoarthritis or spontaneous fusion. Patients with such hips seek treatment and may be considered for total hip replacement, but it is well recognised that recrudescence of infection may occur in such joints even after they have been quiescent for many years. Such recrudescence after an arthroplasty is a major complication and may lead to complete failure (Johnson, Barnes and Owen 1979).

Early reports of the safety of low friction arthroplasty in patients with septic or tuberculous arthritis of the hip which has long been quiescent are encouraging. Hardinge, Cleary and Charnley (1979), from this hospital, reported no infection in 13 previously septic hips and 27 healed tuberculous hips after a mean followup of 32 months. Jupiter et al. (1981) reported one infection in a series of 27 patients with previously septic hips after a mean follow-up of 43 months. In the 24 of these 27 patients whose radiographs were reviewed, six had radiolucency at the bone-cement interface, though all had satisfactory function. There was no recrudescence of infection in seven tuberculous hips given anti-

R. Laforgia, MD, Chir Orthop, Orthopaedic Surgeon

2a Clinica Ortopedica, Universita di Bari, Via Garruba 3, Bari, Italy.

J. C. M. Murphy, FRCS, Consultant Orthopaedic Surgeon

The Hip Centre, Wrightington Hospital, Appley Bridge, Wigan, Lancashire WN6 9EP, England.

T. R. Redfern, MCh Orth, FRCS Ed, Senior Orthopaedic Registrar Department of Orthopaedic Surgery, Royal Liverpool Hospital, Prescott Street, Liverpool L7 8XP, England.

Correspondence should be sent to Dr R. Laforgia.

(C) 1988 British Editorial Society of Bone and Joint Surgery

0301-620X/88/3098 \$2.00

J Bone Joint Surg [Br] 1988;70-B:373-6. tuberculous therapy before operation. However, Kim et al. (1979) reported one case of reactivation of tuberculous infection in a series of 20 patients despite antituberculous treatment before surgery.

Against this background we have reviewed the results of 72 low friction arthroplasties performed in previously tuberculous hips and 42 in 38 patients who had had septic arthritis. Follow-up was for a minimum of two years. We comment on the indications for operation and offer suggestions for the management of these difficult cases.

\section{PATIENTS AND METHODS}

Between 1970 and 1981, a total of 59 low friction arthroplasties were performed on 52 patients who had a past history of haematogenous septic arthritis of the hip. Six patients were excluded from the study because their follow-up was less than two years, four because their records were inadequate and four because the radiographs suggested a different pathology, although a history of sepsis was obtained. The 42 remaining hips in 38 patients were studied.

In the same period, 105 arthroplasties were performed on 99 patients with a past history of tuberculous arthritis of the hip. Eight hips with less than two years follow-up, seven patients with an uncertain history of tuberculosis and six patients whose radiographs suggested a different pathology were excluded. In all, 72 quiescent tuberculous hips in 68 patients were studied. The mean period of follow-up for both groups of patients was five years (range 2 to 17 years).

Patients were grouped according to their symptoms and the radiographic appearance of the hip (Kim et al. 1979). The patients in Group 1 complained of pain on movement of the hip or on weight-bearing. Radiographs 
showed that some joint space was still present although the acetabulum and femoral head were deformed by the previous infection. Patients in Group 2 complained of pain in the low back or knee and were found on radiography to have surgical or spontaneous fusion of the involved hip. All patients were graded clinically by Charnley's modification of the Merle d'Aubigné and Postel classification (Charnley 1972); clinical records were made on standard assessment cards.

Anti-tuberculous medication by rifampicin was used in only 11 of the previously tuberculous hips $(15 \%)$ and was supplemented by isoniazid and ethambutol in one case and by streptomycin in one other. Treatment started three weeks before operation and continued for three months after operation.

A Charnley design of hip prosthesis was used in all cases, and was inserted through a trans-trochanteric approach in a Charnley clean air enclosure with vertical laminar air flow and using body exhaust suits. Eightyeight of the 114 operations were performed by consultants, the remainder by surgeons in training. Bacteriological samples were taken from the capsule, the acetabulum and the medullary canal of the femur in 87 cases but the capsule itself was not excised in any patient. Cement containing gentamicin was used in 32 hips; the other 82 were fixed with CMW cement with no antibiotics.

\section{RESULTS}

\section{Septic arthritis}

There were 38 patients with a mean age at operation of 46 years (range 26 to 70 years). There were 21 women and 17 men, and 22 left hips and 20 right hips were replaced. The mean age at the onset of septic arthritis had been 13 years (range one week to 69 years). The organism responsible for the initial infection was unknown in the majority of patients.

Treatment of the initial infection had lasted for an average of 2.3 years (range 3 months to 9 years). In 20 patients from the pre-antibiotic era it had included bed rest, traction, plaster spica, and drainage or sequestrectomy. The other 18 patients had been treated with antibiotics. In all, 29 hips had had from one to three previous operations (mean 1.4), including drainage, sequestrectomy, Girdlestone pseudarthrosis, intertrochanteric osteotomy and arthrodesis. The average time from the onset of the initial sepsis to the arthroplasty was 32.8 years (range one to 59 years).

Of the 28 hips in Group 1, in which the joint space was preserved but damaged, 17 had unilateral disease, three had bilateral disease and in eight cases the hip problem was only one of the patient's disabilities. The mean pre-operative scores were 3.4 for pain, 3.2 for function and 2.7 for movement. Sixteen patients required sticks or crutches and two were wheelchairbound. After arthroplasty the scores rose to 5.6 for pain,
5.0 for function and 5.1 for movement (Table I). Only five patients still required sticks or crutches and one used a wheelchair.

Of the 14 hips in Group 2 (fused hips), four had unilateral disease, eight were bilaterally affected and in two the hip problem was only part of their disability. The mean pre-operative scores in Group 2 patients were 5.6 for pain, 3.5 for function and 1.0 for movement. Seven patients required sticks or crutches and two used a wheelchair. After arthroplasty the scores rose to 5.7 for

Table I. Results of low friction arthroplasty after old septic arthritis in 42 hips and old tuberculous infection in 72 hips, graded according to Charnley (1972)

\begin{tabular}{|c|c|c|c|c|c|}
\hline & & \multicolumn{2}{|c|}{ Septic arthritis } & \multicolumn{2}{|c|}{ Tuberculous arthritis } \\
\hline & & Pre-op & Post-op & Pre-op & Post-op \\
\hline $\begin{array}{l}\text { Group } 1 \\
\text { (mobile) }\end{array}$ & $\begin{array}{l}\text { Pain } \\
\text { Function } \\
\text { Movement }\end{array}$ & $\begin{array}{l}3.4 \\
3.2 \\
2.7\end{array}$ & $\begin{array}{l}5.6 \\
5.0 \\
5.1\end{array}$ & $\begin{array}{l}3.8 \\
3.3 \\
1.8\end{array}$ & $\begin{array}{l}5.95 \\
5.2 \\
4.6\end{array}$ \\
\hline $\begin{array}{l}\text { Group } 2 \\
\text { (fused) }\end{array}$ & $\begin{array}{l}\text { Pain } \\
\text { Function } \\
\text { Movement }\end{array}$ & $\begin{array}{l}5.6 \\
3.5 \\
1.0\end{array}$ & $\begin{array}{l}5.7 \\
5.0 \\
4.6\end{array}$ & $\begin{array}{l}5.6 \\
3.6 \\
1.0\end{array}$ & $\begin{array}{l}5.7 \\
4.7 \\
4.5\end{array}$ \\
\hline
\end{tabular}

pain, 5.0 for function and 4.6 for movement, and only three patients used sticks or crutches.

Complications. Early postoperative complications developed in seven of the Group 1 patients with mobile hips, including three non-fatal pulmonary emboli, two trochanteric detachments and one urinary tract infection. One wound failed to heal and the patient, after nine visits to the operating theatre for debridement and suture, developed deep infection by a coagulase-negative staphylococcus. The prosthesis was removed.

Late deep sepsis developed in two patients, one due to a coagulase-negative staphylococcus and the other to Staphylococcus pyogenes. One late infection appeared after four years; this hip was revised two years later. The other infection began three years after operation, requiring revision after another three years. In Group 2 patients with fused hips there were three early postoperative complications. One patient developed foot drop, one had an early wound infection which cleared with antibiotics, and in the other patient incomplete wound healing led to deep infection with a coagulase negative staphylococcus. Revision of this arthroplasty was needed after four years.

Bacteriology. Routine bacteriological swabs were taken during most of the operations. A coagulase-negative staphylococcus was cultured in five cases and pseudomonas in one. Deep sepsis did not follow in any of these culture-positive hips although minor radiographic changes are visible in two hips at two years and one at five years. Prophylactic antibiotics had been used for about six weeks to cover 15 of the 42 operations, but there was no fixed regimen and a variety of antibiotics were 
employed. Of the four cases which developed deep infection three had not had antibiotics; the fourth had had Cephradine for six weeks. Antibiotic loaded cement was used in only nine patients, none of whom suffered deep sepsis.

Radiography. In Group 1, 19 hips (68\%) showed no change between the immediate postoperative film and the latest available film taken after an average of five years. Non-progressive demarcation around the socket was seen in two cases (7\%). Three other patients were clinically normal but erosions were seen around the calcar in one, in another the socket appeared to be loose within the cement, while the third had a periosteal reaction around the tip of the femoral stem.

In Group 2 half the cases showed no radiographic change with time, but ectopic calcification was present in four hips and non-progressive demarcation was present at the bone-cement interface of the socket in two hips.

\section{Tuberculous arthritis}

Seventy-two low friction arthroplasties were performed in 68 patients whose mean age at operation was 49 years (range 32 to 75 years). There were 40 women and 28 men with 38 left hip replacements and 34 right. The mean age at the onset of tuberculosis was 7 years 4 months (range 10 months to 22 years).

Most patients had first been treated in the preantibiotic era and only six patients had had a course of streptomycin, the others having been treated with rest, traction, a plaster spica or on a frame. Treatment had lasted an average of four years (range 6 months to 14 years). Ten patients had had a sinus but only one continued to discharge at the time of the hip arthroplasty. Previous operations for 37 hips had included drainage, sequestrectomy, osteotomy, fusion and leg lengthening. Each hip had had from one to four previous operations (mean 1.7). The mean time from the onset of tuberculosis to the hip arthroplasty was $\mathbf{4 0}$ years (range 17 to 72 years).

Before arthroplasty the ESR ranged from 2 to $82 \mathrm{~mm}$ in the first hour (mean 14). Only 13 patients had radiographic evidence of healed pulmonary tuberculosis, but one patient had a classical Pott's kyphosis.

In Group 1, 18 patients had unilateral problems, 11 had bilateral hip involvement and nine had problems other than the hip. Before operation the mean scores were 3.8 for pain, 3.3 for function and 1.8 for movement (Table I). Sixteen patients needed sticks for walking. After arthroplasty the scores became 5.95 for pain, 5.2 for function and $\mathbf{4 . 6}$ for movement and only six patients continued to use sticks.

In Group 2, 11 hips had fused spontaneously and 23 had been arthrodesed. Fourteen were unilateral, 12 had bilateral problems and eight had problems other than the hip. The mean pre-operative scores were 5.6 for pain, 3.6 for function and 1.0 for movement, eight of the patients requiring sticks for walking. After arthroplasty the scores rose to 5.7 for pain, 4.7 for function and 4.5 for movement. Five patients still needed a stick.

Complications. In Group 1 patients the operation was often technically demanding. The femur fractured during reaming in one case and five required acetabular reconstruction. Caseous material was recovered from a cyst in the ilium in one patient and from within the capsule in two others. Acid-fast bacilli were not found in this material. The patient with a discharging sinus at the time of arthroplasty was managed by thorough curettage; two years later there was no evidence of infection or loosening.

Three patients had deep venous thrombosis and one suffered a non-fatal pulmonary embolism. One patient had a foot drop and wound healing was delayed in two. Leg length discrepancies of more than $5 \mathrm{~cm}$ were seen in three patients, all of whom required a shoe raise. Later complications included seven patients who had knee pain caused by valgus or varus deformity, two requiring realignment osteotomy and one a total knee replacement. Three patients complained of low back pain, one of whom needed Harrington instrumentation for correction of a scoliosis.

A deep infection was seen in one patient three years after arthroplasty, with pain, skin inflammation over the hip and an ESR of $96 \mathrm{~mm}$ in the first hour. This resolved with a six-month course of minocycline; two years later the patient remained asymptomatic. One patient developed recrudescence of tuberculous infection 49 years after the first infection. Although early cultures were negative a sinus developed 12 months postoperatively and mycobacteria were cultured from this nine months later.

In Group 2 patients, complications included two cases of deep venous thrombosis, two early trochanteric detachments and seven patients with leg length discrepancies of approximately $2.5 \mathrm{~cm}$. Two patients suffered knee pain and one had persisting low back pain.

In this group no case of recrudescence of tuberculosis was seen but deep infections developed in two patients. One patient developed a pseudomonas infection six years after arthroplasty and required revision; the other had had four operations prior to the arthroplasty, developed a staphylococcal infection and eventually required removal of the prosthesis.

Bacteriology. Bacteriological swabs were obtained from 57 hips, with only nine positive results: a coagulasenegative staphylococcus from six, a streptococcus from two and a klebsiella from one. None of these hips developed loosening or infection. Prophylactic antibiotic cover was provided for only nine hips, using a variety of antibiotics.

Radiography. In 24 patients in Group $1(63 \%)$ there was no difference between the immediate postoperative and the most recent film. Demarcation around both components was seen in the patient who developed recrudescent tuberculosis, and in two other asymptomatic patients. 
Abnormal appearances were seen in 11 other patients: three had loosening of the socket, two had sinking of the stem and fractures of the femoral cement, three had periosteal reactions around the tip of the stem, and socket wear without loosening was seen in two. One hip showed massive ectopic new bone formation which led to spontaneous extra-articular fusion.

In Group 2, radiographic signs of loosening were seen in nine hips with sinking of the stem in seven, loosening of the stem in one and socket loosening in one. All these patients were symptom free. Socket wear was seen in two patients, ectopic calcification in one and a periosteal reaction at the tip of the stem in one.

\section{DISCUSSION}

Recrudescence of tuberculosis complicated only one of 72 arthroplasties in old tubercular hips, a rate of $1.4 \%$. This rate is lower than the $5 \%$ reported by $\mathrm{Kim}$ et al. (1979) but Hardinge et al. (1979) and Jupiter et al. (1981) reported no such complication. Our patient showed no specific features which would have allowed prediction of this problem, though as in the five other reported cases of recrudescence, the joint space of the hip had been preserved. Recrudescence after operation on hips with a sound arthrodesis has not been reported.

We believe that all patients with previous tuberculous infection who are offered hip arthroplasty should be given a pre-operative course of anti-tuberculous drugs, unless it is known that they have already had such treatment. Patients with a mobile joint must be considered to be at a greater risk of recrudescence. Three arthroplasties for old quiescent tuberculous arthritis were complicated by deep infection. The rate of $4.2 \%$ is comparable to the $3.7 \%$ reported by Dupont and Charnley (1972) for low friction arthroplasty undertaken after the failure of previous operations and we believe that the underlying cause in our cases was the multiple previous operations.

In 42 patients with a history of old septic arthritis, low friction arthroplasty was complicated by deep infection in four $(9.5 \%)$. This rate is higher than those reported by Dupont and Charnley (1972) and by Jupiter et al. (1981). By contrast with the results for old tuberculous arthritis, the number of previous operations did not appear to influence the final result of the arthroplasty; the four cases which were complicated by deep infection had not had a larger number of previous operations. It seems that other factors must be responsible.

The infection rate did not appear to be influenced by the age at which the primary infection occurred or by the length and nature of the treatment at that stage. The preoperative ESR was not of prognostic value. Three of the four patients had not been given prophylactic antibiotic cover, and none had antibiotic-loaded cement. Infection had occurred in four of 33 hips with no antibiotic in the cement; there were no infections in nine hips where Palacos-R cement with added gentamicin was used.

The incidence of deep infection tended to be less in hips in which the previous septic arthritis or its treatment had resulted in arthrodesis (one of 14 hips), than in hips with a distorted joint space (three of 28 hips). The deep sepsis rate was lower when a consultant performed the operation (two of 34 hips) than when it was carried out by a surgeon in training (two of 8 hips).

We recommend that hip arthroplasty for patients with old quiescent septic arthritis should be covered by prophylactic antibiotics, that antibiotic-loaded cement should be used, and that these difficult operations should by undertaken only by experienced surgeons.

\section{REFERENCES}

Charnley J. The long-term results of low-friction arthroplasty of the hip performed as a primary intervention. J Bone Joint Surg [Br] 1972;54-B:61-76.

Dupont JA, Chamby J. Low-friction arthroplasty of the hip for the failures of previous operations. J Bone Joint Surg [Br] 1972;54-B:77-87.

Hardinge K, Cleary J, Charnley J. Low-friction arthroplasty for healed septic and tuberculous arthritis. J Bone Joint Surg [Br] 1979;61-B:144-7.

Johnson R, Barnes KL, Owen R. Reactivation of tuberculosis after total hip replacement. J Bone Joint Surg [Br] 1979;61-B:148-50.

Jupiter JB, Karchmer AW, Lowell JD, Harris WH. Total hip arthroplasty in the treatment of adult hips with current or quiescent sepsis. J Bone Joint Surg [Am] 1981;63-A:194-200.

Kim YY, Ko CU, Lee SW, Kwak BM. Replacement arthroplasty using the Charnley prosthesis in old tuberculosis of the hip. Int Orthop 1979;3:81-8. 